\title{
The relationship between brain and liver damage in chronic alcoholic patients
}

\author{
W ACKER,* EJ APS, $\dagger$ SISIR K MAJUMDAR, $¥$ GK SHAW,§ \\ ALLAN D THOMSON\|!
}

From the Department of Neuropsychiatry, Institute of Psychiatry, ${ }^{*}$ London, Queen Mary's Hospital, Sidcup $\dagger$ Bexley Hospital, Bexley, and the Gastroenterology \& Liver Unit, Greenwich District Hospital, London, $\ddagger$ Elmdene Alcoholic Unit, Bexley Hospital, Bexley,§ and the Gastroenterology \& Liver Unit, Greenwich District Hospital, $\|$ London, $U K$

SUMMARY CT scan measures of topographical brain changes, liver status as determined by biopsy, and clinical factors were studied in a group of detoxified chronic alcoholic patients. It was found that greater topographical brain changes were associated with greater severity of liver disease.

Brain damage is an important complication of alcohol abuse but little is known of the mechanisms of injury or its natural history. There is evidence that brain damage may result from interference with the supply of nutrients and that this may be a consequence of liver injury. ${ }^{12}$ To clarify the relationship between liver disease and topographical brain changes, a group of chronic alcoholic patients was studied by clinical examination, computed tomography of the head and by liver biopsy. The data were examined for associations between brain changes, liver damage, age and length of drinking history.

\section{Patients and methods}

\section{Patients}

A clinical interview and physical examination of each patient was carried out as reported in detail by Lishman et al. ${ }^{3}$ Patients were excluded if their history included factors other than alcoholism, associated with cerebral damage (for example older than 65 years, head injury resulting in posttraumatic amnesia of longer than 24 hours, epilepsy since childhood or prolonged coma) or if there was a history of major psychiatric illness or if physical examination revealed obvious clinical signs of organic brain disease. The number of years during which patients admitted to drinking more than $150 \mathrm{~g}$ ethanol daily at least twice a week was taken as an index of the length of drinking history. The patients studied were consecutive admissions to an alcoholic rehabilitation and detoxification facility who, for clinical reasons, were referred for liver biopsy. Of the 41 patients studied, 39 were male.

Address for reprint requests: Dr GK Shaw, Elmdene Alcoholic Unit, Bexley Hospital, Bexley, Kent DA5 2BW, UK.

Received 10 June 1982

Accepted 10 July 1982

\section{Methods}

Liver biopsy was performed in each patient using a Trucut needle and histological studies were reported without knowledge of the clinical, biochemical or neuroradiological details. On the basis of hepatic histology patients were divided into four groups-those with normal livers, those with steatonecrosis, those with hepatitis, and those with cirrhosis. Computed tomographic head scans (CT scans) were carried out on each patient at the Department of Neuroradiology, the Maudsley Hospital, using an EMI CT1010 Scanner. Details of the scanning procedures have been given by Lishman et al ${ }^{3}$ From photographic records of the scans, measurements were obtained of the relative size of the ventricular system (ventricle/brain ratio- $\mathrm{V} / \mathrm{B}$ ratio), the width of the cortical sulci, the width of the Sylvian fissures, and the width of the interhemispheric fissure. The V/B ratio was determined by planimetric measurement of the inner circumference of the skull and the outer limits of the ventricular system in those two CT scan cuts in which the ventricular system was judged to be most extensive. The $\mathrm{V} / \mathrm{B}$ ratio used in the statistical analysis was the mean of measures made by two independent assessors. Three measures of cortical status (sulcal width, Sylvian fissure width, and interhemispheric fissure width) were determined by visual inspection of CT records. These were then compared to norms defining the limits of different categories. In the case of sulcal width and interhemispheric fissure width there were two categories: (1) normal or minimum enlargement and (2) moderate or pronounced enlargement. In the case of Sylvian fissure width there were three categories: (1) normal width; (2) moderate enlargement; (3) pronounced enlargement. These ratings of sulcal width, Sylvian fissure width and interhemispheric fissure width were made by two independent assessors. In cases of disagreement, the more conservative (that is more normal) rating was adopted. In all cases CT scans were delayed until patients had been free from alcohol. for two weeks and drug free for one week. Liver biopsy was carried out within 10 days of admission, usually between days 5 and 10 . In all cases the raters 
reported their results without knowledge of the clinical and liver biopsy details of patients.

\section{Statistical analysis used}

The data collected were either continuous variables or categorical variables. In the case of continuous variables (age, length of drinking history and ventricle/brain ratio) group differences between patients in different liver disease categories were tested for significance using analyses of variance. Analyses of variance were carried out in two ways: (1) across four levels of liver disease, normal, steatonecrosis, hepatitis and cirrhosis and (2) across two levels of liver disease, mild or $\rightarrow$ liver disease (normal and steatonecrosis groups conbined) and severe liver disease (hepatitis and cirrhosis group combined). In the case of categorical variables (sulcal width, Sylvian fissure width and interhemispheric width) tests for associations between categories of liver disease, brain changes and age were used. For this purpose patients were divided into three age groups: 40 years and younger; $41-50$ years; and 51 years and older. In each circumstance tests for associations between the effects of liver disease and age on brain changes as assessed by CT scan were always considered simultaneously. In the case of tests for differences in the means of variables this was done by partialling out the effect of age on CT brain changes. In the case of tests for associations between categorical variables three dimensional frequency tables were analysed by fitting log-linear and linear-logistic models. These may be thought of as analogous to the usual linear models employed in the analysis of variance of continuous data (see Everitt, Ch 5 for details). ${ }^{4}$

\section{Results}

A summary of all variables collected is listed in table 1 .

\section{Analyses of variance on continuous variables}

Age-an analysis of variance indicated no significant differences in mean age between patients in the four categories of liver disease $(F(3,37)=1 \cdot 473, p=0.238)$. Equally there was no significant difference in mean age between the mild and severe liver disease groups $(t(40)=1.053, \mathrm{p}=0.135)$.

Length of drinking history-an analysis of variance indicated no significant differences in mean length of drinking history between patients in the four categories of liver disease after partialling out the effect of age $(F(3,36)=0.365, p=0.778)$. Equally there was no significant difference in mean length of drinking history between the mild and severe liver disease groups $(t(40)=0.797, p=0.436)$.

CT scan measures-an analysis of variance indicated that there were significant differences in mean V/B ratios for patients in the four liver disease categories $(\Sigma(3,37)=2 \cdot 88, p=0 \cdot 048)$.

When age was entered as a co-variate, analysis indicated that the differences in mean V/B ratios for the four liver disease groups were enhanced by the addition of age as a co-variate $(F(3,36)=4 \cdot 399, p=0 \cdot 005)$. In this analysis of variance/co-variance the mean differences in V/B ratios attributable to liver disease category were not significant $(F(3,36)=1 \cdot 727, p=$ $0 \cdot 179$ ), but the mean differences in $V / B$ ratios attributable to the co-variate age, were significant $(F(1,36)=$ $7 \cdot 431, p=0 \cdot 01$ ).

A two-way analysis of variance was also carried out. In this two-way analysis, one independent variable was age at three levels- -40 years and younger, $41-50$ years, and 51 years and older. The second independent variable was liver disease at two levels-mild and severe. This analysis indicated that there was a significant combined effect of age and liver disease on the $\mathrm{V} / \mathrm{B}$ ratios $(F(3,35)=3 \cdot 382, p=0.029)$. The effect of liver disease

Table 1 Summary table: Age, years drinking and measures of brain damage distributed by categories of liver damage

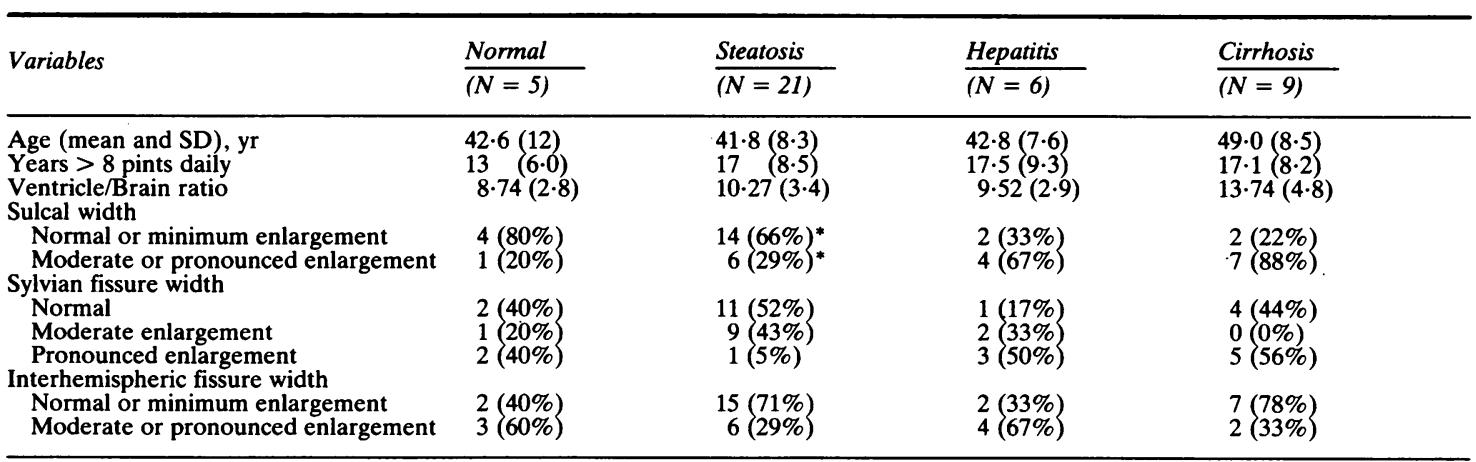

*'There was one case, a patient with steatonecrotic changes, in whom measurement of sulcal width was not possible.

In the cases of age, length of drinking history and V/B ratios group means are given with standard deviation in parentheses. In the cases of sulcal width, Sylvian fissure width and interhemispheric width the absolute number of patients in each group is given with percentages in parentheses. 
on the V/B ratios was not significant $(F(1,35)=1 \cdot 328$, $\mathrm{p}=0 \cdot 257$ ), but the effect of age on the $\mathrm{V} / \mathrm{B}$ ratios was significant $(\mathrm{F}(2,35)=3 \cdot 505, \mathrm{p}=0.041)$.

\section{Analysis of categorical variables via log-linear and linear-logistic models}

In each of these analyses the relationship between CT scan categorical variables and age and CT scan categorical variables and liver disease were considered simultaneously. Separate analyses were carried out for sulcal width, Sylvian fissure width and interhemispheric fissure width. To increase cell size for these analyses, liver disease categories were collapsed into two groups (mild or no liver disease and severe liver disease) and age was converted into a categorical variable (40 years and younger, $41-50$ years, and 51 years and older). Linear-logistic models were fitted to the data to assess the effects of liver disease and age on the categorical CT scan measures, and to determine whether or not these effects were additive.

The three-way table of liver disease category $\times$ age $x$ sulcal width is given in table 2 . Analysis of the data indicates that there is a significant association between liver disease category and sulcal width (chi-squared $1 \mathrm{df}$ $=8 \cdot 01, \mathrm{p}<0 \cdot 01$ ), but there is no significant association between age category and sulcal width (chi-squared 2 $\mathrm{df}=1 \cdot 21, \mathrm{p}<0 \cdot 30$ ).

A three-way table of liver disease category $x$ age $x$ Sylvian fissure width is given in table 3 . Analysis of the data indicated that there is a significant association between liver disease category and Sylvian fissure width (chi-squared $2 \mathrm{df}=8.88, \mathrm{p}<0.02$ ). No significant association between age category and Sylvian

Table 2 Sulcal width $\times$ age $\times$ liver disease

Table of sulcal width at two levels $(0-$ normal and minimal sulcal enlargement and 1 -moderate and pronounced sulcal enlargement), by age at three levels $(<=40 \mathrm{yr}, 41-50 \mathrm{yr},>$ $51 \mathrm{yr})$, by liver disease at two levels ( 0 -normal and steatosis, and 1 -cirrhosis and hepatitis)

\begin{tabular}{llllllll}
\hline & Age & $<=40 y r$ & $41-50$ & $y r$ & $>$ \\
& Liver & 0 & 1 & 0 & 1 & 0 & 1 \\
Sulcal width & 0 & 8 & 1 & 6 & 3 & 4 & 0 \\
& 1 & 4 & 2 & 1 & 5 & 2 & 4
\end{tabular}

Models fitted in log-linear analysis-sulcal width

$\begin{array}{lll}\text { Models } & \text { Likelihood ratios } & D F \\ \text { Liver and age no effect } & 11.99 \mathrm{~L} 1 & 5 \mathrm{df} 1 \\ \text { Liver effect only } & 3.98 \mathrm{~L} 2 & 4 \mathrm{df} 2 \\ \text { Age effect only } & 10.78 \mathrm{~L} 3 & 3 \mathrm{df} 3\end{array}$

Computation of chi 2-sulcal width

\begin{tabular}{llll}
$\begin{array}{l}\text { Association of liver } \\
\text { disease and sulcal width }\end{array}$ & $\begin{array}{l}\text { Chi } 2 \\
\mathrm{~L} 1-\mathrm{L} 2\end{array}$ & $\begin{array}{l}\text { DF } \\
\text { df1-df2 } 2\end{array}$ & $\begin{array}{l}\text { Probability } \\
\mathrm{p}<0.01\end{array}$ \\
$\begin{array}{l}\text { Association of age and } \\
\mathrm{L} 1-\mathrm{L} 3\end{array}$ & $\begin{array}{l}5-4=1 \\
\text { df1-df3 }\end{array}$ & $\mathrm{p}<0.30$ \\
sulcal width & $11.99-10.78=1.21$ & $5-3=2$ & $\mathrm{p}<0.30$ \\
\hline
\end{tabular}

Table 3 Sylvian fissure width $\times$ age $\times$ liver disease

Table of Sylvian fissure width at three levels $(0$-normal, 1 -moderate enlargement, and 2-pronounced enlargement), by age at three levels ( $<=40 \mathrm{yr}, 41-50 \mathrm{yr},>51 \mathrm{yr})$, by liver disease at two levels ( 0 -normal and steatosis, and $1-$ cirrhosis and hepatitis)

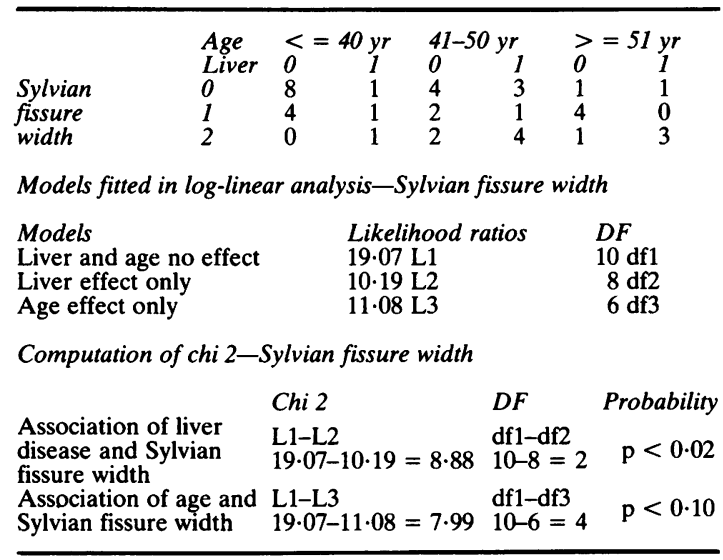

Table 4 Interhemispheric fissure width $\times$ age $\times$ liver disease

Table of interhemispheric fissure width at two levels ( 0 normal and minimum enlargement, and 1 -moderate and pronounced enlargement), by age at three levels $(<=40 \mathrm{yr}$, $41-50 \mathrm{yr},>51 \mathrm{yr})$, by liver disease at two levels $(0-$ normal and steatosis, and $1-$ cirrhosis and hepatitis)

\begin{tabular}{|c|c|c|c|c|}
\hline & Age & $<=40 y r$ & $41-50 y r$ & $>=51 y r$ \\
\hline \multirow{3}{*}{ IHF width } & Liver & 0 & 0 & 0 \\
\hline & 0 & 11 & 5 & 1 \\
\hline & 1 & 1 & 3 & 5 \\
\hline
\end{tabular}

Models fitted in log-linear analysis-interhemispheric fissure width

Models

Liver and age no effect

Liver effect only

Age effect only

Computation of chi 2-interhemispheric fissure width

\begin{tabular}{llll}
$\begin{array}{l}\text { Association of liver } \\
\text { disease and interhemi- } \\
\text { spheric fissure width }\end{array}$ & $\begin{array}{l}\text { Chi } 2 \\
\mathrm{~L} 1-\mathrm{L} 2\end{array}$ & $\begin{array}{l}\text { DF } \\
\mathrm{df1}-\mathrm{df} 2\end{array}$ & Probability \\
$\begin{array}{l}\text { Association of age and } \\
\text { interhemispheric } \\
\text { fissure width }\end{array}$ & $\mathrm{L} 1-\mathrm{L} 3$ & $\mathrm{1} 3-45=0.12$ & $5-4=1 \mathrm{p}<0.80$ \\
\hline
\end{tabular}

fissure width was demonstrated (chi-squared 4 df = 7.99, $\mathrm{p}<0 \cdot 10$ ).

Analysis of the interhemispheric fissure width data (table 4) indicated that there is no significant association between liver disease category and interhemispheric fissure width (chi-squared $1 \mathrm{df}=0 \cdot 12$, $\mathrm{p}<0 \cdot 80$ ). There is a significant association between 
age category and interhemispheric fissure width (chisquared $2 \mathrm{df}=6.68, \mathrm{p}<0.05)$.

In each of these analyses it was found necessary to include a term for the interactive effect of liver disease and age on the categorical CT scan measure.

\section{Discussion}

Although cirrhotic alcoholics tended to be older, analysis of variance did not indicate any significant difference in age between the four liver disease groups. Similarly, although alcoholics with normal livers tended to have shorter drinking histories, the differences in length of drinking history between the four liver disease groups were not statistically significant. Equally, when the liver disease groups were collapsed into two larger groups, mild or no liver disease and severe liver disease, no significant difference in either age or length of drinking history was noted. It must be borne in mind, however, that the numbers are small in some of the liver disease categories and thus genuine differences in either age or length of drinking histories may be obscured.

The analysis of variance in V/B ratios across liver disease categories reveals the unsatisfactory matching for age. Although the mean V/B ratio is more than $50 \%$ larger in the cirrhotic patients than in patients with normal livers and some $30 \%$ larger in cirrhotic patients than in patients with steatonecrosis and hepatitis, the cirrhotic patients were also older than the other patient groups. Therefore, when age is considered as a co-variate and liver disease category as an independent factor, age effects obscure any effects which may have been attributable to liver disease.

The present data indicate that the association of age with ventricular enlargement is more important than the association of liver disease with ventricular enlargement. Peculiarities of the present data suggest that a further study with a better age match across the liver disease groups would be important.

The analyses of categorical variables in which age, liver disease, and CT scan measures were considered simultaneously revealed statistically significant associations between liver disease and measures of sulcal width and between liver disease and measures of Sylvian fissure width. Age was not significantly associated either with measures of sulcal width or with measures of Sylvian fissure width. There was, however, a tendency for age to be associated with measures of Sylvian fissure width, and there was a significant association between age and measures of interhemispheric width. Measures of interhemispheric width were not significantly associated with liver disease category.

Whilst it would be premature to regard these results as conclusive they differ from those reported by Lee $e t$ $a l^{1}$ in that a significant association between severity of liver disease and topographical brain changes has been demonstrated. Since this association is independent of age, it might suggest that certain individuals are predisposed both to liver injury and to brain injury on prolonged exposure to alcohol, whilst other individuals are relatively resistant to such physical changes. Alternatively, it might be argued that whereas alcohol-related brain damage occurs independently of the effect of alcohol on the liver, in the later stages of the disease the metabolic consequences of a deranged liver augment the leleterious effect of alcohol on the brain. It might therefore be of interest to determine if women, who reportedly show more liver damage than men, also show more evidences of brain changes. Similarly, it would be of interest to study the brain topography of a group of middle-aged patients with non-alcohol related liver disease.

The present investigation indicates that in chronic alcoholics topographical brain changes become more pronounced as the severity of liver injury increases.

The authors thank Professor WA Lishman and Dr Maria Ron from the Institute of Psychiatry who read the scans, and Mr BS Everitt, Head of the Biometrics Unit, Institute of Psychiatry, who gave statistical advice and assistance. We also thank Mrs Jean Johns for secretarial and typing assistance.

\section{References}

${ }^{1}$ Lee K, Moller L, Hardt F, Haubek A, Jensen E. Alcoholinduced brain damage and liver damage in young males. Lancet 1979;2:759-61.

${ }^{2}$ Lusins J, Zimberg S, Smokler H, Gurley K. Alcoholism and cerebral atrophy: a study of $\{50$ patients : with $: C T$ scan' and psychological testing. Alcoholism: Clinical and Ex perimental Research 1980;4: 406-11.

${ }^{3}$ Lishman WA, Ron MA, Acker W. Computed tomography of the brain and psychometric assessment of alcoholic patients-a British study. In: Richter D, ed. Addiction and Brain Damage. London: Croom Helm, 1980;215-27.

${ }^{4}$ Everitt BS. The Analysis of Contingency Tables. London: Chapman \& Hall, 1977. 